Metaanalyse Schlaganfall

\title{
Lysetherapie für über 80ig Jährige
}

Die Erfolgsrate der Lysetherapie ist bei älteren Patienten keineswegs schlechter.

Je früher die sogenannte Lysetherapie einsetzt, desto besser sind die Behandlungsergebnisse für die Patienten - auch über 80ig Jährige. Dies geht aus einer Metaanalyse [1] hervor, die jetzt in der Fachzeitschrift "The Lancet ${ }^{\prime}$ erschienen ist.

Die Untersuchung bestätigte, dass die Lysebehandlung im Zeitfenster von 4,5 h nach dem Schlaganfall und auch bei älteren Menschen effektiv ist, erklären die Deutsche Schlaganfall-Gesellschaft (DSG) und die Deutsche Gesellschaft für Neurologie (DGN).

Die Wirksamkeit der Lysetherapie wurde vor fast 20 Jahren erstmals in einer klinischen Studie belegt, trotz- dem werde die Therapie mit dem Thrombolytikum Alteplase (rt-PA) nach Angaben der Fachgesellschaften nur bei etwas $10 \%$ der Schlaganfallpatienten in Deutschland durchgeführt. Denn nur etwa 30-40\% der Schlaganfallpatienten erreichen rechtzeitig die Klinik.

Für die Metaanalyse wertete ein internationales Forscherteam neun Studien mit insgesamt 6756 Patienten aus. Die Chancen eines Patienten, den Schlaganfall ohne schwere Behinderungen zu überleben, waren in den ersten $3 \mathrm{~h}$ um $75 \%$ höher als in der Vergleichsgruppe, die keine Lysetherapie erhielt. Wurde die Lyse 3-4,5 h nach dem Schlaganfall begonnen, betrug der Vorteil noch $26 \%$. Die Ergebnisse bestätigen den Effekt der Lyse im Zeitfenster von 4,5 h.

Da 1729 Teilnehmer der Studienteilnehmer älter als 80 Jahre waren, liefert die aktuelle Metaanalyse hier erstmals zuverlässige Ergebnisse. Die Erfolgsrate der Lysetherapie war bei Hochbetagten keineswegs schlechter, die Ergebnisse waren tendenziell sogar besser.

psychopraxis.neuropraxis $2015 \cdot 18: 30$ DOI 10.1007/s00739-015-0235-4 Online publiziert: 28. Januar 2015 c) Springer-Verlag Wien 2015 